\title{
Tumor Results Vendor Name
}

National Cancer Institute

\section{Source}

National Cancer Institute. Tumor Results Vendor Name. NCI Thesaurus. Code C117415.

The literal identifier of the vendor or laboratory that performs a tumor results assessment. 